\title{
TWO ASPECTS OF COLOR SUPERCONDUCTIVITY: GAUGE INDEPENDENCE AND NEUTRALITY
}

\author{
A. GERHOLD* \\ Institut für Theoretische Physik, Technische Universität Wien, \\ Wiedner Hauptstr. 8-10, A-1040 Vienna, Austria \\ E-mail: gerhold@hep.itp.tuwien.ac.at
}

\begin{abstract}
A formal proof is given that the fermionic quasiparticle dispersion laws in a color superconductor are gauge independent. It is shown that the gluon (photon) field acquires a non-vanishing expectation value in a color superconductor, which is related to color (electric) neutrality.
\end{abstract}

\section{Introduction}

It is well known that cold dense quark matter is a color superconductor. ${ }^{1}$ Color superconducting phases are characterized by a non-vanishing gap function, which can be calculated self-consistently from the SchwingerDyson equation (gap equation). At asymptotic densities, calculations from first principles (QCD) should be possible. However, calculations of the gap function seem to give different results for covariant and Coulomb gauge. ${ }^{2}$ This was the motivation to construct a formal proof of gauge independence, which is presented in Sec. 2. It turns out that this proof involves nonvanishing expectation values of the gauge fields. These expectation values act as effective chemical potentials for color and electric charge, which will be briefly discussed in Secs. 3-5.

*Work supported by the Austrian Science Foundation FWF, project no. 16387-N08. 


\section{Gauge independence of fermionic quasiparticle dispersion laws}

The inverse quark propagator in the Nambu-Gor'kov basis is given by

$$
\begin{aligned}
\mathcal{S}^{-1} & =\left(\begin{array}{cc}
\phi+\mu \gamma_{0}+\Sigma & \Phi^{-} \\
\Phi^{+} & \not-\mu \gamma_{0}+\bar{\Sigma}
\end{array}\right) \\
& =\left.\int d^{4} x e^{-i Q \cdot x} \frac{\delta^{2} \Gamma}{\delta \bar{\Psi}(x) \delta \Psi(0)}\right|_{\psi=\bar{\psi}=A_{i}^{a}=0, A_{0}^{a}=\tilde{A}_{0}^{a}},
\end{aligned}
$$

where $\Phi^{+}$is the gap function, $\Phi^{-}(Q)=\gamma_{0}\left[\Phi^{+}(Q)\right]^{\dagger} \gamma_{0}, \Sigma$ is the quark self energy, $\bar{\Sigma}(Q)=C[\Sigma(-Q)]^{T} C^{-1}$ with the charge conjugation matrix $C, \Gamma$ is the effective action, and $\tilde{A}$ is the expectation value of the gluon field. The change in the effective action under the variation of some gauge parameter(s) is given by the following gauge dependence identity, ${ }^{3,4}$

$$
\delta \Gamma=\Gamma_{, i} \delta X^{i}+\int d x \frac{\delta \Gamma}{\delta A^{a \mu}(x)} \delta X_{(A)}^{a \mu}(x) .
$$

We use the DeWitt notation for the fermions, $i=\left(\psi(x), \psi_{c}(x)\right)^{T}, \bar{i}=$ $\left(\bar{\psi}(x), \bar{\psi}_{c}(x)\right)$. The $\delta X$ 's in Eq. (2) are quite complicated functionals, whose diagrammatic expansion is shown in Ref. 4. After taking the second derivative of Eq. (2), switching to momentum space, ${ }^{\mathrm{a}}$ and taking the determinant with respect to Nambu-Gor'kov, Dirac, color and flavor indices, we obtain ${ }^{6}$

$$
\delta \operatorname{det}\left(\Gamma_{i \bar{j}}\right)+\delta \tilde{A}^{a 0} \frac{\partial}{\partial \tilde{A}^{a 0}} \operatorname{det}\left(\Gamma_{i \bar{j}}\right)=-\operatorname{det}\left(\Gamma_{i \bar{j}}\right)\left[\delta X_{, k}^{k}+\delta X_{, \bar{k}}^{\bar{k}}\right] .
$$

This identity means that the total variation of the determinant of the inverse propagator is proportional to the determinant of the inverse propagator itself. Therefore the fermionic quasiparticle dispersion laws are gauge independent, provided that the singularities of $\delta X$ do not coincide with those of the quark propagator. In principle $\delta X$ could develop mass shell singularities, as it is the case for the fermion damping rate in high temperature QCD. ${ }^{7}$ Then a gauge independent result could only be obtained by introducing an IR cutoff, to be lifted only at the very end of the calculation. ${ }^{8}$ However, in the case of color superconductivity a simple IR cutoff turns out not to be sufficient to remove the gauge dependence of the gap in covariant gauges. Indeed, it has been argued that this gauge dependence will only disappear after the inclusion of vertex corrections in the gap equation. ${ }^{9}$

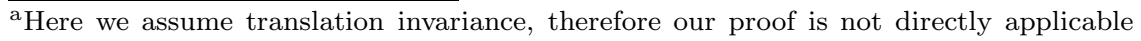
for inhomogeneous color superconducting phases, which might appear at intermediate densities, see e.g. Ref. 5.
} 


\section{Gluon tadpole and color neutrality}

In contrast to NJL models, where color neutrality has to be imposed as an external condition, ${ }^{10}$ the color superconducting phases in full QCD are automatically color neutral. This can be demonstrated in the following way. ${ }^{6,11}$ The fields $A_{0}^{a}$ appear in the QCD action as Lagrange multipliers for the Gauss law constraint (at least in a gauge which does not involve $A_{0}^{a}$.) Therefore in the path integral the integration over the zeromomentum modes $A_{0, \vec{p}=0}^{a}$ produces delta functions, $\delta\left(N_{a}\right)$, where $N_{a}$ are the color charges. This means that only color neutral field configurations contribute to the partition function.

In a color superconductor the gluon field acquires a non-vanishing expectation value. ${ }^{6,12,13}$ The leading order contribution to this expectation value can be computed from the one-loop gluon tadpole diagram. For the 2SC phase we find ${ }^{6}$

$$
\mathcal{T}^{a} \simeq-\delta^{a 8} \frac{2 g}{\sqrt{3} \pi^{2}} \mu \phi^{2} \ln \left(\frac{\phi}{2 \mu}\right) .
$$

The expectation value of $A_{0}^{a}$ can be obtained by attaching the external gluon propagator, which gives ${ }^{6,13}$

$$
\tilde{A}_{0}^{8} \sim \phi^{2} /\left(g^{2} \mu\right) .
$$

This expectation value acts as an effective chemical potential for the color charge (via $\mu_{8}=g A_{0}^{8}$ ), thus ensuring color neutrality. ${ }^{13}$

In Eq. (4) we have computed the quantity

$$
\left.\mathcal{T}^{a} \sim \frac{\delta \Gamma}{\delta A_{0}^{a}}\right|_{A=0} .
$$

We remark that at the order of our computation this calculation is com-

pletely equivalent to the self-consistent approach of Ref. 13, where $\tilde{A}$ has been computed from the Yang-Mills equation

$$
\left.\frac{\delta \Gamma}{\delta A_{0}^{a}}\right|_{A=\tilde{A}}=0 .
$$

The connection between the two approaches can be seen from the Taylor expansion

$$
\left.\frac{\delta \Gamma}{\delta A_{0}^{a}}\right|_{A=0}=\left.\frac{\delta \Gamma}{\delta A_{0}^{a}}\right|_{A=\tilde{A}}-\left.\frac{\delta^{2} \Gamma}{\delta A_{0}^{a} \delta A_{0}^{b}}\right|_{A=\tilde{A}} \tilde{A}_{0}^{b}+\ldots
$$

On the right hand side the first term vanishes, while the second term is essentially the inverse gluon propagator at zero momentum times the expectation value of $A$. 


\section{Gluon tadpole for CFL with $m_{s} \neq 0$}

In the CFL phase one finds $\tilde{A}_{0}^{a}=0$ at one-loop order for $m_{s}=0$. For finite $m_{s}$ the quark propagator in the Nambu-Gor'kov basis is given by

$$
\mathcal{S}=\left(\begin{array}{cc}
{\left[G_{0}^{-}\right]^{-1}} & \Phi^{-} \\
\Phi^{+} & {\left[G_{0}^{+}\right]^{-1}}
\end{array}\right)^{-1}
$$

with

$$
\left[\left(G_{0}^{ \pm}\right)^{-1}\right]_{r s}^{i j}=\left[\left(\phi \pm \mu \gamma_{0}\right)\left(\delta_{r s}-\delta_{r 3} \delta_{s 3}\right)+\left(\not \pm \mu \gamma_{0}-m_{s}\right) \delta_{r 3} \delta_{s 3}\right] \delta^{i j},
$$

where the lower indices are flavor indices and the upper indices are color indices. At finite $m_{s}$ the Dirac structure of the gap is more complicated than in the massless case, ${ }^{14}$ but for $m_{s} \ll \mu$ we may assume that the additional terms are suppressed at least with $m_{s} / \mu,{ }^{15}$

$$
\left[\Phi^{+}\right]_{r s}^{i j} \simeq \phi_{+} \Lambda_{\mathbf{q}}^{+} \gamma_{5}\left(\delta_{r}^{i} \delta_{s}^{j}-\delta_{r}^{j} \delta_{s}^{i}\right)+\mathcal{O}\left(\frac{m_{s}}{\mu}\right) .
$$

Assuming $m_{s} \gg \phi_{+}$we find for the tadpole diagram at leading order in $m_{s}$

$$
\begin{aligned}
\mathcal{T}^{a} \simeq \delta^{a 8} \frac{g m_{s}^{2}}{2 \sqrt{3} \pi^{3}} \int_{-\infty}^{\infty} d q_{4} \int_{0}^{\infty} d q q \phi_{+}^{2} \\
\quad \times \frac{q_{4}^{2}-3(q-\mu)^{2}-8 \phi_{+}^{2}}{\left(q_{4}^{2}+(q-\mu)^{2}+\phi_{+}^{2}\right)^{2}\left(q_{4}^{2}+(q-\mu)^{2}+4 \phi_{+}^{2}\right)} \\
\simeq \delta^{a 8} \frac{g \mu m_{s}^{2}}{18 \sqrt{3} \pi^{2}}(-21+8 \ln 2),
\end{aligned}
$$

where we have neglected the momentum dependence of the gap function. Attaching the gluon propagator with the Debye mass ${ }^{16}$ proportional to $g \mu$ we find as in Ref. 12

$$
\tilde{A}_{0}^{a} \sim \delta^{a 8} \frac{m_{s}^{2}}{g \mu} .
$$

Therefore the effective chemical potential is $\mu_{8} \sim m_{s}^{2} / \mu$, which is consistent with results from NJL model calculations. ${ }^{10}$

\section{Photon tadpole and electric neutrality}

If one takes into account also electrons and photons, electric neutrality will we achieved by a mechanism which is completely analogous to the one described in Sec. 3. For the expectation value of the photon field one finds in the $2 \mathrm{SC}$ phase (for $m_{s}=0$ )

$$
A^{0} \sim \frac{\phi^{2}}{e \mu} \ln \left(\frac{\phi}{2 \mu}\right) .
$$




\section{Acknowledgements}

I would like to thank C. Manuel and A. Rebhan for very helpful discussions.

\section{References}

1. D. Bailin and A. Love, Phys. Rept. 107, 325 (1984); M. G. Alford, K. Rajagopal and F. Wilczek, Phys. Lett. B422, 247 (1998); R. Rapp, T. Schäfer, E. V. Shuryak and M. Velkovsky, Phys. Rev. Lett. 81, 53 (1998); K. Rajagopal and F. Wilczek, The condensed matter physics of QCD, hep-ph/0011333; M. G. Alford, Ann. Rev. Nucl. Part. Sci. 51, 131 (2001).

2. D. T. Son, Phys. Rev. D59, 094019 (1999); R. D. Pisarski and D. H. Rischke, Phys. Rev. D61, 074017 (2000); T. Schäfer and F. Wilczek, Phys. Rev. D60, 114033 (1999); D. K. Hong, V. A. Miransky, I. A. Shovkovy and L. C. R. Wijewardhana, Phys. Rev. D61, 056001 (2000) [Erratum: ibid. D62, 059903 (2000)]; S. D. H. Hsu and M. Schwetz, Nucl. Phys. B572, 211 (2000); W. E. Brown, J. T. Liu and H.-c. Ren, Phys. Rev. D61, 114012 (2000); K. Rajagopal and E. Shuster, Phys. Rev. D62, 085007 (2000); R. D. Pisarski and D. H. Rischke, Nucl. Phys. A702, 177 (2002); D. K. Hong, T. Lee, D. P. Min, D. Seo and C. Song, Phys. Lett. B565, 153 (2003).

3. B. W. Lee and J. Zinn-Justin, Phys. Rev. D7, 1049 (1973); R. Fukuda and T. Kugo, Phys. Rev. D13, 3469 (1976). N. K. Nielsen, Nucl. Phys. B101, 173 (1975); I. J. R. Aitchison and C. M. Fraser, Ann. Phys. 156, 1 (1984).

4. R. Kobes, G. Kunstatter and A. Rebhan, Phys. Rev. Lett. 64, 2992 (1990); R. Kobes, G. Kunstatter and A. Rebhan, Nucl. Phys. B355, 1 (1991); A. Rebhan, Lect. Notes Phys. 583, 161 (2002).

5. M. G. Alford, J. A. Bowers and K. Rajagopal, Phys. Rev. D63, 074016 (2001); R. Casalbuoni and G. Nardulli, Rev. Mod. Phys. 76, 263 (2004).

6. A. Gerhold and A. Rebhan, Phys. Rev. D68, 011502 (2003).

7. R. Baier, G. Kunstatter and D. Schiff, Phys. Rev. D45, 4381 (1992).

8. A. Rebhan, Phys. Rev. D46, 4779 (1992).

9. D. f. Hou, Q. Wang and D. H. Rischke, Phys. Rev. D69, 071501 (2004).

10. P. Amore, M. C. Birse, J. A. McGovern and N. R. Walet, Phys. Rev. D65, 074005 (2002); M. Alford and K. Rajagopal, JHEP 0206, 031 (2002);

A. W. Steiner, S. Reddy and M. Prakash, Phys. Rev. D66, 094007 (2002); I. Shovkovy and M. Huang, Phys. Lett. B564, 205 (2003); M. Huang and I. Shovkovy, Neutral dense quark matter, hep-ph/0311155.

11. S. Y. Khlebnikov and M. E. Shaposhnikov, Phys. Lett. B387, 817 (1996).

12. A. Kryjevski, Phys. Rev. D68, 074008 (2003).

13. D. D. Dietrich and D. H. Rischke, Prog. Part. Nucl. Phys. 53, 305 (2004).

14. R. D. Pisarski and D. H. Rischke, Phys. Rev. D60, 094013 (1999).

15. T. D. Fugleberg, Phys. Rev. D67, 034013 (2003).

16. D. H. Rischke, Phys. Rev. D62, 054017 (2000). 